\title{
Obstructive sleep apnoea and urine catecholamines in hypertensive males: a population-based study
}

\author{
A. Elmasry*,\#, E. Lindberg*, J. Hedner", C. Janson*, G. Boman*
}

\begin{abstract}
Obstructive sleep apnoea and urine catecholamines in hypertensive males: a populationbased study. A. Elmasry, E. Lindberg, J. Hedner, C. Janson, G. Boman. (C)ERS Journals Ltd 2002.

ABSTRACT: Studies addressing the relationship between obstructive sleep apnoea (OSA) and sympathoadrenal activity have been criticized for poor control of factors known to confound sympathetic function, including hypertension. The aim of this study was to investigate the relationship between OSA and urinary catecholamines in a population-based sample of hypertensive males.

In 1994, 2,668 males aged 40-79 yrs answered a questionnaire regarding sleep disorders and somatic diseases. Of those who reported hypertension, an age-stratified sample of 116 was selected for monitoring of breathing during sleep and overnight urine analysis.

Subjects with OSA, defined as apnoea-hypopnoea index $\geqslant 10 \cdot h^{-1}$, had higher concentrations of urinary normetanephrine $\left(182 \pm 57\right.$ versus $141 \pm 45 \mu \mathrm{mol} \cdot \mathrm{mol}^{-1}$ creatinine, $p<0.001)$ and metanephrine $\left(70 \pm 28\right.$ versus $61 \pm 28 \mu \mathrm{mol} \cdot \mathrm{mol}^{-1}$ creatinine, $\mathbf{p}<0.05$ ) in comparison to subjects without OSA. In a multiple regression analysis, there was an association between variables of sleep-disordered breathing and normetanephrine and metanephrine concentrations, independent of major confounding factors.

The authors concluded that, in a population-based sample of hypertensive males, obstructive sleep apnoea is associated with increased urinary concentrations of extraneuronal metabolites of catecholamines independent of major confounding factors, suggesting increased sympathoadrenal activity. Elevated sympathoadrenal activity may explain the increased cardiovascular morbidity associated with obstructive sleep apnoea.
\end{abstract}

Eur Respir J 2002; 19: 511-517.
*Dept of Medical Sciences, Respiratory Medicine and Allergology, Uppsala University, Akademiska sjukhuset, Uppsala, Sweden. " Dept of Chest Diseases, Ain Shams University, Cairo, Egypt. "Depts of Pulmonary Medicine and Clinical Pharmacology, Sahlgrenska University Hospital, Gothenburg, Sweden.

Correspondence: E. Lindberg, Dept of Medical Sciences, Respiratory Medicine and Allergology, Akademiska Sjukhuset, S-75185 Uppsala, Sweden.

Fax: 46186112819

E-mail: eva.lindberg@medsci.uu.se

Keywords: Catecholamines

hypertension

population-based

sleep apnoea

Received: December 222000

Accepted after revision October 26 2001

This work was supported by the Swedish Heart and Lung Foundation.
Increased sympathetic activity has been widely demonstrated in patients with obstructive sleep apnoea (OSA) [1-4]. Altered sympathetic activity has been considered as the main link in the possible causeeffect relationship between OSA and hypertension, both in animal $[5,6]$ and in human studies [1, 7]. A similar mechanism has also been considered as an explanation of the association between OSA and other cardiovascular diseases, including cardiac dysrhythmia, angina pectoris, myocardial infarction, congestive heart failure, and cerebrovascular disease [8-11]. Moreover, this altered sympathetic activity was corrected by continuous positive airway pressure treatment $[12,13]$.

Small sample size and inadequate correction for factors that may confound the sympathetic activity, such as age, weight and medication have been criticized in most studies of OSA and sympathetic activity [14]. The correction for the known association between hypertension and sympathetic alteration $[15,16]$ is also inadequate in most of these studies. Apart from the study of JENNUM et al. [17] on self-reported snoring and plasma norepinephrine in the elderly, no general population-based study of the association between OSA and sympathetic activity could be found.
The aim of the present study was to investigate the possible relationship between sleep-disordered breathing and sympathetic activity, as assessed by measuring urinary catecholamines, in a population-based sample of hypertensive males in the age range 40-79 yrs.

\section{Subjects and methods}

\section{Population}

In 1984, a postal questionnaire was answered by 3,201 males, randomly selected from the total male population in the age group 30-69 yrs in the city of Uppsala in Sweden (response rate $79.6 \%$ ). Ten years later, 226 had died and another questionnaire was sent to the remaining 2,975 subjects. The second questionnaire in 1994 was answered by 2,668 subjects $(89.7 \%)$ [18].

Hypertension was defined as reporting regular medical check-ups for hypertension and/or answering "Yes" to the question "Do you have high blood pressure?" in the 1994 questionnaire, and also receiving treatment with antihypertensive medication(s). Accordingly, there were a total of $392(14.7 \%)$ 
hypertensive subjects. The present analysis is based upon an age-stratified sample of 116 of these hypertensive subjects. All these subjects took part in a casecontrol study with the main purpose of evaluating the prevalence of OSA in hypertensives and normotensive controls [19]. The reason why only hypertensives were included in the present analysis was that they were selected from the general population whereas the normotensive controls were selected to match the hypertensives in age and body mass index (BMI). Age stratification with 10-yr age strata was performed and hypertensives were included randomly within each age stratum. This led to 116 hypertensive subjects distributed as follows: $25(21.6 \%)$ between $40-49 \mathrm{yrs}$, $39(33.6 \%)$ between $50-59$ yrs, $31(26.7 \%)$ between 60-69 yrs, and $21(18.1 \%)$ between $70-79$ yrs.

\section{Study design}

Investigative procedures relevant to this study were performed on two successive days. On day one, the subject arrived in the clinic for an interview by an experienced research nurse and received the recording equipment for home monitoring of breathing during sleep as well as detailed instructions for use. Subjects were instructed to void urine before going to bed and then to collect the urine during the night and the next morning in a clean flask. In the morning following the sleep recording, weight, height, waist and hip circumferences were measured by the same nurse for all the subjects. The urine flask was delivered to the nurse. The overnight recording was transferred to a computer for analysis of respiratory events.

\section{Anthropomorphic measurements}

BMI was calculated as the weight in $\mathrm{kg}$ divided by the height in $\mathrm{m}^{2}$. Waist circumference was measured midway between the lower rib margin and the anterior superior iliac spine. Hip circumference was measured at the widest circumference over the greater trochanters. The waist-to-hip ratio (WHR) was calculated.

\section{Urine analysis}

$\mathrm{HCl}(15 \mathrm{~mL}$ of $6 \mathrm{M})$ was added to the clean flask before urine collection. After homogenization aliquots of $100 \mathrm{~mL}$ were transferred to clean test tubes and frozen at $-20^{\circ} \mathrm{C}$. Samples were analysed for catecholamines using high performance liquid chromatography with electrochemical detection [20]. Quantified catecholamines and metabolites were norepinephrine, alpha-(aminomethyl) vanillyl alcohol (3-methylnorepinephrine) (normetanephrine), alpha-(methylaminomethyl) vanillyl alcohol (3-methylepinephrine) (metanephrine), (3-Methoxy tyramine) (mettyramine) and 3-methoxy 4-hydroxy mandelic acid (MHMA), also called Vanilmandelic acid. The results were expressed in $\mu \mathrm{mol} \cdot \mathrm{mol}^{-1}$ of creatinine except for MHMA (mmol $\mathrm{mol} \cdot \mathrm{mol}^{-1}$ of creatinine).

\section{Hypertension and cardiovascular disease}

On day one, subjects were asked the duration of their diagnosis of hypertension, how long they had been treated with antihypertensive $\operatorname{drug}(\mathrm{s})$, and which $\operatorname{drug}(s)$ they were receiving. The reported drugs were classified as beta $(\beta)$-blockers, angiotensin-convertingenzyme (ACE) inhibitors, calcium channel antagonists and others. Duration of hypertension was defined as time in yrs since the subject received the diagnosis of hypertension. The number of antihypertensive drugs received was used to measure the severity of hypertension and the participants were classified here as those taking one drug, two drugs and more than two drugs.

The participants were also asked if they ever had been referred to hospital or were under regular medical suspension for angina pectoris, myocardial infarction, heart failure and/or stroke. Cardiovascular disease (CVD) was defined as answering "yes" to at least one of these questions. Blood pressure was measured by the research nurse in the morning after the night recording.

\section{Night study}

Whole night respiratory monitoring was performed using the Eden Trace II multichannel recording system (model 3711; Eden Tec corporation, Eden Prairie, MN, USA). This system records snoring sounds by a microphone, oronasal airflow by a thermistor, breathing movements by an impedance belt, body position, and oxygen saturation by a finger probe [21].

\section{Analysis of night study}

All respiratory events were scored automatically (ETS 2.0; E INFINITI Medical, Täby, Sweden) and manually edited by one of the investigators, blinded to the actual patient identity.

Total sleep time (TST) was estimated by visual assessment of the overnight tracing in conjunction with the subject's diary [21, 22]. All the periods of irregularities, artefacts or disconnections were rejected. As a minimum TST of $4 \mathrm{~h}$ was required, 14 records were rejected after the first night but accepted after a second night study. A desaturation event was defined as a fall in oxygen saturation of $\geqslant 4 \%$. An apnoea was defined as cessation of oronasal airflow for at least $10 \mathrm{~s}$. A hypopnoea was defined as a reduction in oronasal airflow of $\geqslant 50 \%$ of the average peak airflow during the preceding $2 \mathrm{~min}$ for at least $10 \mathrm{~s}$, followed by a desaturation and/or an increase in thoraco-abdominal impedance of $\geqslant 50 \%$. Apnoea/ hypopnoea index (AHI) and desaturation index (DI) were calculated as the total number of such events divided by TST. Average oxygen saturation (average $\mathrm{S}_{\mathrm{a}, \mathrm{O}_{2}}$ ) and minimum oxygen saturation (min $\mathrm{Sa}_{\mathrm{a}} \mathrm{O}_{2}$ ) were determined. Snoring sounds were scored automatically, and snoring index was defined as the percentage of TST occupied by sounds $\geqslant 90$ decibels 
[22]. The informed consent of all participants was obtained and the study was approved by the Ethics Committee of the Medical faculty at Uppsala University.

\section{Statistics}

Statistical analyses were performed using the Stat View SE+Graphics ${ }^{\mathrm{TM}}$ (C) 1988 Abacus Concepts, Inc. Berkeley, CA, USA). To achieve a normal distribution all continuous variables were log transformed. Groups were compared using the unpaired t-test for continuous variables, and Chi-squared test for proportions. Linear regression analysis was used to calculate correlations between continuous variables and the results were presented as standard coefficient of regression (r) while Spearman Rank correlation was used for nominal variables and the results were presented as rho value. A p-value $<0.05$ was regarded as statistically significant.

\section{Results}

In the questionnaire on day one of the study, 110 subjects reported the duration of their hypertension diagnosis (mean \pm SD of $13.2 \pm 10.1$ yrs). $\beta$-blockers were taken by 59 subjects, 43 used ACE inhibitors, and calcium channel antagonists were used by 39 subjects while other antihypertensive drugs (vasodilators or

Table 1.-Anthropometric data, urine catecholamines, blood pressure, antihypertensive drugs, and prevalence of cardiovascular disease (CVD) in subjects with obstructive sleep apnoea (OSA) compared to subjects without OSA (non-OSA)

\begin{tabular}{lcc}
\hline & Non-OSA & OSA \\
\hline Subjects n & 72 & 44 \\
Age yrs & $58.9 \pm 9.7$ & $63.8 \pm 8.8^{* *}$ \\
BMI kg $\cdot \mathrm{m}^{-2}$ & $26.7 \pm 3.7$ & $29.2 \pm 4.8^{* *}$ \\
WHR & $1.0 \pm 0.03$ & $1.01 \pm 0.03$ \\
Overnight urine volume & $555 \pm 304$ & $634 \pm 343$ \\
U-norepinephrine $\mu \mathrm{mol} \cdot \mathrm{mol}^{-1}$ & $18.2 \pm 8.6$ & $20.2 \pm 6.8$ \\
U-normetanephrine $\mu \mathrm{mol}^{-1} \mathrm{~mol}^{-1}$ & $141 \pm 45$ & $182 \pm 57^{* * *}$ \\
U-MHMA mmol $\cdot \mathrm{mol}^{-1}$ & $1.7 \pm 0.7$ & $1.7 \pm 0.5$ \\
U-metanephrine $\mu \mathrm{mol} \cdot \mathrm{mol}^{-1}$ & $61 \pm 28$ & $70 \pm 28^{*}$ \\
U-mettyramine $\mu \mathrm{mol} \cdot \mathrm{mol}^{-1}$ & $91 \pm 61$ & $94 \pm 31$ \\
Systolic blood pressure $\mathrm{mmHg}^{-}$ & $148 \pm 17.6$ & $153 \pm 20.4$ \\
Diastolic blood pressure $\mathrm{mmHg}^{-}$ & $91 \pm 9.4$ & $92 \pm 12.5$ \\
Users of $\beta$-blockers & $40(55.6)$ & $19(43.2)$ \\
Users of ACE inhibitors & $27(37.5)$ & $16(36.4)$ \\
Users of calcium channel & $24(33.3)$ & $15(34.1)$ \\
$\quad$ antagonists & $10(13.9)$ & $16(36.4)^{* *}$ \\
CVD &
\end{tabular}

Data are presented as mean \pm SD or $\mathrm{n}(\%)$. BMI: body mass index; WHR: waist-to-hip ratio; U: urinary; MHMA: methoxy-hydroxy-mandelic acid; ACE: angiotensinconverting enzyme. Non-OSA was defined as apnoea/ hypopnoea index $<10 \cdot \mathrm{h}^{-1}$ of sleep and OSA as apnoea/ hypopnoea index $\geqslant 10 \cdot \mathrm{h}^{-1}$ of sleep. Catecholamines were measured per mol of creatinine. *: $\mathrm{p}<0.05 ; * *: \mathrm{p}<0.01$; $* * *: \mathrm{p}<0.001$ diuretics) were used by 29 subjects. Overall, 58 subjects were receiving one drug, 40 were receiving two drugs and 11 were receiving $>$ two drugs. Twenty-six subjects reported 39 CVD events, with 20 angina pectoris, 11 myocardial infarction, four heart failure, and four cerebrovascular stroke.

\section{Catecholamines and obstructive sleep apnoea}

Subjects were separated into two groups based on their AHI: AHI $\geqslant 10 \cdot h^{-1} \quad(O S A, n=44)$ and AHI $<10 \cdot \mathrm{h}^{-1}$ (non-OSA, $\mathrm{n}=72$ ). The OSA subjects were older and more obese. Neither systolic nor diastolic blood pressure was significantly different between the two groups. The frequency of use of the different antihypertensive drugs did not differ significantly between the two groups. CVD was, however, significantly more prevalent in subjects with OSA. Urine catecholamines were generally higher in the OSA subjects although significance was reached only for normetanephrine and metanephrine (table 1).

The 75th percentile for each analysed catecholamine was used as a cut-off point to divide the 116 subjects into those with high, and those with low concentrations. Subjects with high normetanephrine concentration $(n=30)$ had a significantly higher prevalence of OSA when compared with those with a low level $(\mathrm{p}=0.0005)$. The differences between the groups were not significant for the other catecholamines (fig. 1). Similar differences remained if the 90th percentile was used (data not shown).

\section{Univariate linear regression}

Urinary normetanephrine was significantly associated with AHI, DI, min $S_{\mathrm{a}}, \mathrm{O}_{2}$ and average $\mathrm{Sa}_{\mathrm{a}} \mathrm{O}_{2}$

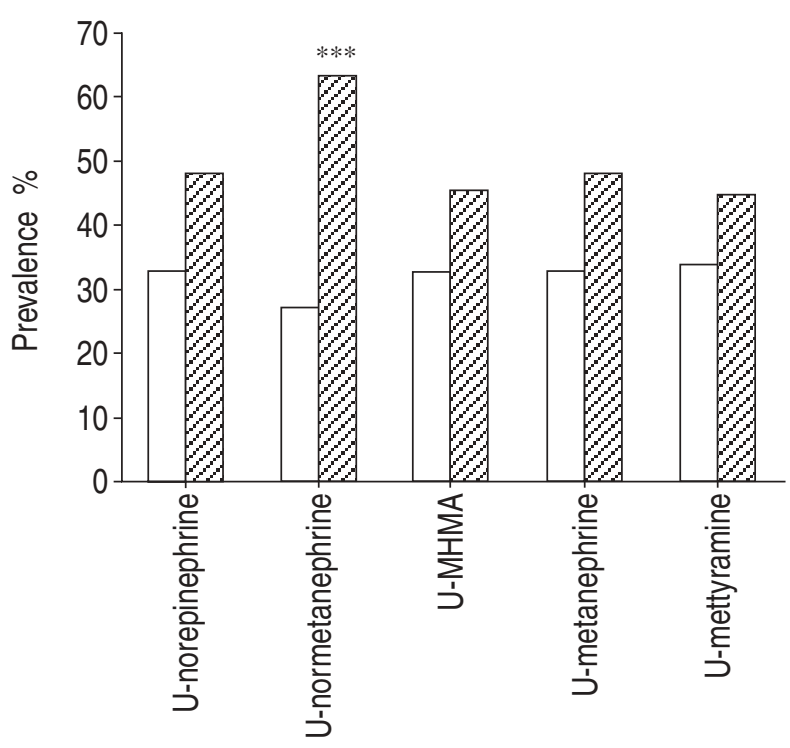

Fig. 1.- Prevalence of obstructive sleep apnoea in subjects with the highest percentile $\left(\mathbb{Z}: \geqslant 75 \mathrm{th}^{\%}\right.$ ) in comparison to all others $(\square$ : $<75$ th $\%$ ) for every urine catecholamine. U: urinary; MHMA: methoxy-hydroxy-mandelic acid. All catecholamines are measured per mol of creatinine. ${ }^{* * *}: \mathrm{p}<0.001$. 
Table 2.-Relationships between sleep-disordered breathing parameters and catecholamines in urine in simple linear regression analysis

\begin{tabular}{lccccc}
\hline & AHI & DI & Min $S_{\mathrm{a}, \mathrm{O}_{2}}$ & Average $S_{\mathrm{a}, \mathrm{O}_{2}}$ & Snoring index \\
\hline U-norepinephrine $\mu \mathrm{mol} \cdot \mathrm{mol}^{-1}$ & 0.09 & 0.08 & -0.13 & $-0.19^{*}$ & 0.004 \\
U-normetanephrine $\mu \mathrm{mol} \cdot \mathrm{mol}^{-1}$ & $0.33^{* * *}$ & $0.34^{* * *}$ & $-0.29^{* *}$ & $-0.29^{* *}$ & -0.02 \\
U-MHMA mmol $\cdot \mathrm{mol}^{-1}$ & -0.01 & -0.03 & 0.09 & 0.09 & $-0.19^{*}$ \\
U-metanephrine $\mu \mathrm{mol} \cdot \mathrm{mol}^{-1}$ & 0.15 & 0.16 & -0.05 & -0.06 & -0.16 \\
U-mettyramine $\mu \mathrm{mol} \cdot \mathrm{mol}^{-1}$ & 0.07 & 0.05 & -0.09 & -0.18 & 0.10 \\
\hline
\end{tabular}

Data are presented as standard coefficient of regression (r). AHI: apnoea/hypopnoea index; DI: desaturation index; Min $\mathrm{Sa}_{\mathrm{a}} \mathrm{O}_{2}$ : minimum arterial oxygen saturation during night; Average $\mathrm{Sa}_{\mathrm{a}} \mathrm{O}_{2}$ : average arterial oxygen saturation during night; $\mathrm{U}$ : urinary; MHMA: methoxy-hydroxy-mandelic acid. Catecholamines were measured per mol of creatinine. ${ }^{*}: \mathrm{p}<0.05$; $* *: \mathrm{p}<0.01 ; * * *: \mathrm{p}<0.001$

(table 2). Urinary norepinephrine was associated with average $\mathrm{Sa}_{\mathrm{a}} \mathrm{O}_{2}$, while MHMA was inversely associated with snoring index. Metanephrine and mettyramine were not significantly correlated with the markers studied.

All catecholamines were significantly related to age, with the highest correlations for normetanephrine $(\mathrm{r}=0.46, \mathrm{p}<0.001)$ and metanephrine $(\mathrm{r}=0.43, \mathrm{p}<0.001)$. BMI was inversely associated with metanephrine $(\mathrm{r}=-0.29, \mathrm{p}<0.01)$ and WHR was inversely associated with norepinephrine $(\mathrm{r}=0.2, \mathrm{p}<0.05)$.

Table 3.-Concentrations of urinary catecholamines in hypertensive subjects with reported cardiovascular disease (CVD) in comparison to hypertensive subjects without CVD

\begin{tabular}{|c|c|c|}
\hline & $\begin{array}{l}\text { Subjects without } \\
\text { CVD }\end{array}$ & $\begin{array}{l}\text { Subjects with } \\
\text { CVD }\end{array}$ \\
\hline Subjects n & 90 & 26 \\
\hline $\begin{array}{l}\text { U-norepinephrine } \\
\mu \mathrm{mol} \cdot \mathrm{mol}^{-1}\end{array}$ & $19 \pm 8.3$ & $18.8 \pm 7$ \\
\hline $\begin{array}{l}\text { U-normetanephrine } \\
\mu \mathrm{mol} \cdot \mathrm{mol}^{-1}\end{array}$ & $149 \pm 49$ & $181 \pm 60^{* *}$ \\
\hline $\begin{array}{l}\mathrm{U}-\mathrm{MHMA} \\
\mathrm{mmol} \cdot \mathrm{mol}^{-1}\end{array}$ & $1.6 \pm 0.7$ & $1.8 \pm 0.5$ \\
\hline $\begin{array}{l}\text { U-metanephrine } \\
\mu \mathrm{mol} \cdot \mathrm{mol}^{-1}\end{array}$ & $60 \pm 24$ & $80 \pm 36^{* *}$ \\
\hline $\begin{array}{l}\text { U-mettyramine } \\
\mu \mathrm{mol} \cdot \mathrm{mol}^{-1}\end{array}$ & $91 \pm 56$ & $96 \pm 30$ \\
\hline
\end{tabular}

\section{Catecholamines and severity of hypertension}

As expected, considering the lack of adjustment for current medication, there was no correlation between systolic or diastolic blood pressure and any of the catecholamines. Moreover, the number of antihypertensive drugs prescribed was not significantly related to any of the catecholamines. However, the duration of hypertension was significantly correlated with normetanephrine $(\mathrm{r}=0.25, \mathrm{p}<0.01)$ and norepinephrine $(\mathrm{r}=0.24, \mathrm{p}<0.01)$.

Subjects with reported CVD $(n=26)$ had significantly higher concentrations of normetanephrine and metanephrine in comparison to those without CVD $(n=90$; table 3$)$.

\section{Multiple linear regression}

The relationship between parameters of sleepdisordered breathing and urinary catecholamines was investigated in a multivariate regression model, after adjustment for age, BMI and parameters of severity of hypertension. In that model, normetanephrine was significantly associated with AHI, DI, min $S \mathrm{a}_{1} \mathrm{O}_{2}$ and average $S \mathrm{a}_{1} \mathrm{O}_{2}$ and metanephrine was significantly associated with AHI and DI (table 4).

\section{Relationships among urinary catecholamines}

There was a significant positive relationship between norepinephrine and normetanephrine $(\mathrm{p}<0.001)$ but not between norepinephrine and MHMA ( $\mathrm{p}=0.16)$ (fig. 2). Moreover, there was an association between

Table 4.-Multiple linear regression analysis for sleep-disordered breathing parameters (one at a time) and catecholamines in urine after adjustment for age, body mass index, duration of hypertension, severity of hypertension and cardiovascular disease

\begin{tabular}{lccccc}
\hline & AHI & DI & Min $S_{\mathrm{a}, \mathrm{O}_{2}}$ & Average $S_{\mathrm{a}, \mathrm{O}_{2}}$ & Snoring index \\
\hline U-norepinephrine $\mu \mathrm{mol} \cdot \mathrm{mol}^{-1}$ & 0.15 & 0.18 & -0.19 & -0.18 & 0.10 \\
U-normetanephrine $\mu \mathrm{mol} \cdot \mathrm{mol}^{-1}$ & $0.23^{*}$ & $0.34^{* * *}$ & $-0.29^{* *}$ & $-0.25^{* *}$ & 0.08 \\
U-MHMA mmol $\cdot \mathrm{mol}^{-1}$ & -0.13 & -0.16 & 0.19 & 0.16 & -0.14 \\
U-metanephrine $\mu \mathrm{mol} \cdot \mathrm{mol}^{-1}$ & $0.22^{*}$ & $0.27^{* *}$ & -0.13 & -0.08 & 0.02 \\
U-mettyramine $\mu \mathrm{mol} \cdot \mathrm{mol}^{-1}$ & 0.02 & 0.02 & -0.10 & -0.18 & 0.18 \\
\hline
\end{tabular}

Data are presented as partial regression coefficient (r). AHI: apnoea/hypopnoea index; DI: desaturation index; Min $S_{\mathrm{a}} \mathrm{O}_{2}$ : minimum arterial oxygen saturation during night; Average $S \mathrm{a}, \mathrm{O}_{2}$ : average arterial oxygen saturation during night; $\mathrm{U}$ : urinary; MHMA: methoxy-hydroxy-mandelic acid. Catecholamines were measured per mol of creatinine. ${ }^{*}: \mathrm{p}<0.05 ; * *: \mathrm{p}<0.01$; $* * *: \mathrm{p}<0.001$. 

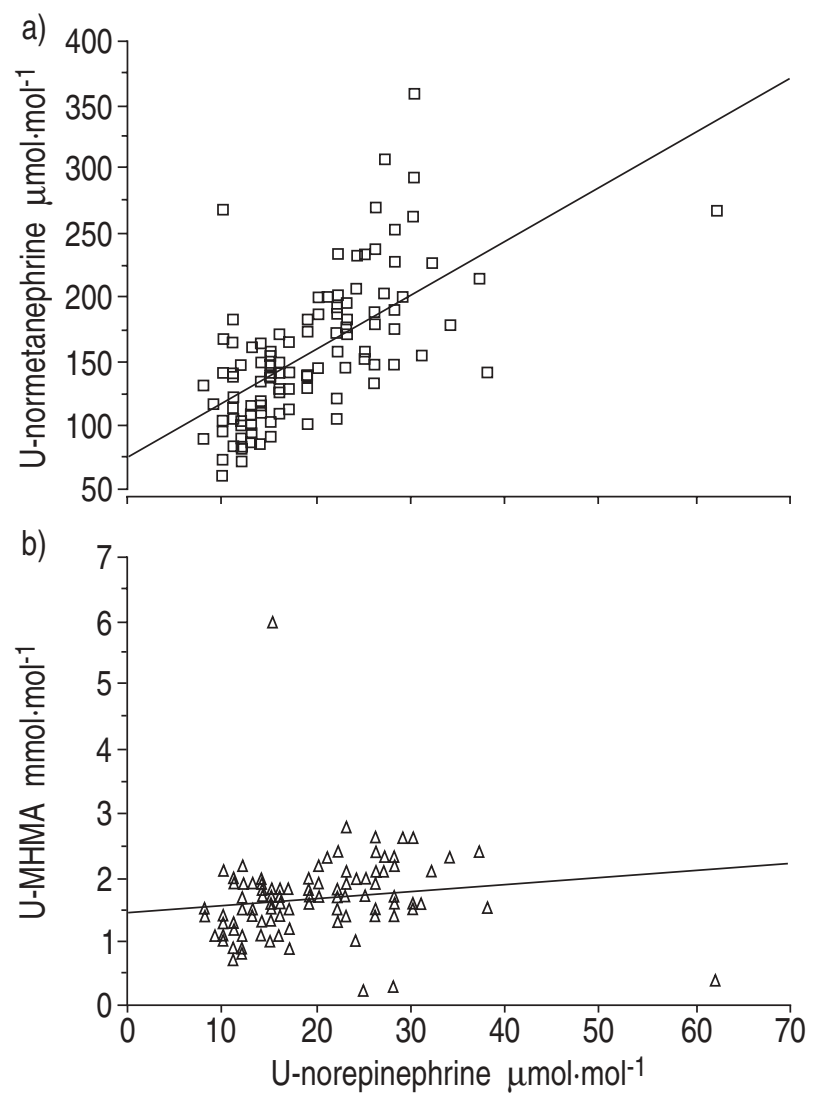

Fig. 2.-Relations between a) urinary (U) norepinephrine and U-normetanephrine (regression coefficient $(\mathrm{r})=0.64 ; \mathrm{p}=0.0001)$ and $\mathrm{b}$ ) U-norepinephrine and U-methoxy-hydroxy-mandelic acid (MHMA; $\mathrm{r}=0.13 ; \mathrm{p}=0.16$ ).

normetanephrine and metanephrine $(\mathrm{r}=0.53, \mathrm{p}<0.001)$, a weak but significant relationship between normetanephrine and MHMA $(\mathrm{r}=0.23, \mathrm{p}<0.05)$ but no significant association between metanephrine and MHMA ( $\mathrm{r}=0.14, \mathrm{p}=0.13)$.

\section{Discussion}

The important finding of the present study is that in a population-based sample of hypertensive males, disordered breathing during sleep was associated with higher urinary excretion of the extraneuronal metabolites, normetanephrine and metanephrine, independent of factors known to confound the metabolism of catecholamines. The finding suggests an increased activity of the sympathetic neurones as well as an increased activity of the adrenal gland. This may be caused by the respiratory events in OSA, particularly hypoxia.

To the best of the authors' knowledge, this is the first population-based study with objective recording of OSA and metabolites of catecholamines. Coy et al. [14] reviewed 24 studies concerning OSA and sympathetic activity. Based on the adequacy of control for the eight variables, hypertension, medications, age, weight, collection of catecholamines, definition of OSA, diet and sample size, that confound catecholamine concentrations, they gave a design index of $0-8$ for each study. The mean design index score was 3.6 with only $16 \%$ of the studies controlling for the effect of hypertension [14]. Although a better correction for confounders was performed in some recent studies [1, 7, 23], the small sample size, the usual selection of severe OSA subjects and inadequate recognition of the effect of hypertension are still common. In the present study, the authors corrected for most of these confounders.

The independent association between sleep breathing disturbances and urinary-normetanephrine has several implications. First, normetanephrine is the O-methylated product of norepinephrine resulting from catechol-O-methyltransferase (COMT) activity, primarily in the synaptic cleft of the sympathetic nerve terminals $[24,25]$. An increased concentration indicates an increase of norepinephrine release and metabolism at nerve terminals. This is further supported by the strong correlation $(\mathrm{r}=0.64)$ between norepinephrine and normetanephrine in the results presented here. Second, about $40-45 \%$ of plasma normetanephrine is derived from metabolism of norepinephrine within the adrenals [25, 26]. Indeed, the adrenal contribution is evidenced by the regresion line for the relationship between norepinephrine and normetanephrine which intersected the ordinate above the origin. By comparing the ordinate-intercept value for normetanephrine $\left(75 \mu \mathrm{mol} \cdot \mathrm{mol}^{-1}\right)$ with mean U-normetanephrine concentration $\left(156 \mu \mathrm{mol} \cdot \mathrm{mol}^{-1}\right)$, a $48 \%$ contribution of the adrenals to U-normetanephrine may be estimated.

The independent association between the sleep breathing disturbances and U-metanephrine has other implications. First, metanephrine is the O-methylated product of epinephrine by COMT [24]. Epinephrine is exclusively formed by the adrenals and almost $97 \%$ of plasma metanephrine is derived from that epinephrine [25]. Consequently, the results indicate an increased activity of the adrenal medulla, possibly as a result of apnoea- and hypopnoea-induced hypoxia or sleep fragmentation. This is further supported by the strong correlation between normetanephrine and metanephrine. Second, although most of the previous reports did not find a significant relationship between plasma or U-epinephrine and OSA $[13,14,23]$, the authors do not think that this conflicts with the observed significant relationship between U-metanephrine and OSA in this study. Almost $90 \%$ of metanephrine in plasma is derived from metabolism of epinephrine before its release into the circulation [25].

While most previous studies found a significant association between OSA and plasma or U-norepinephrine $[7,14]$ the present results were in line with one previous study [12] and showed only a nonsignificant trend towards an association between sleep-disordered breathing and U-norepinephrine. There may be explanations for this discrepancy. All previous investigations were case-control studies based on clinical populations and most of them dealt with small homogenous groups of severe OSA patients. Moreover, some involved nonhypertensive subjects as a control group or failed to correct for the effect of hypertension. Thus, the authors believe that the 
significant relationship between U-normetanephrine and sleep-disordered breathing is a result of increased formation, release and metabolism of norepinephrine.

It is not surprising that there was no relationship between sleep-disordered breathing and MHMA concentration, in spite of its role as a major excretory product of catecholamines. MHMA is produced from catecholamines (mainly norepinephrine) by a process which involves primarily the monoamine oxidase (MAO) enzyme which is located chiefly within the sympathetic nerve terminals [24]. This means a very weak dependence of MAO metabolites, such as MHMA, on the release of norepinephrine, in contrast to COMT metabolites, such as normetanephrine [25]. In the present study, there was no significant correlation between U-norepinephrine and U-MHMA. Another MAO metabolite, dihyroxyphenylglycol remained unchanged in patients with hypertension, angina pectoris or renal artery stenosis, in contrast to norepinephrine and normetanephrine which increased significantly [25].

Different indices of hypoxia appeared to be strong stimuli for sympathoadrenal excitation in OSA $[23,27]$. Other factors such as arousals and sleep fragmentation also may have played a role [7, 28]. As polysomnography was not used in this study, the potential influence of these factors remains unknown.

CVD was defined only by questionnaire-based data. However, the significant association between CVD and OSA, as well as between CVD and normetanephrine and metanephrine, suggest that OSAmediated sympathoexcitation may be an important mechanism underlying increased CVD morbidity in hypertensive subjects. In the present study, BMI showed no major influence on urinary catecholamines. This is in accordance to NARKIEwICZ et al. [29] who found that obesity alone, in the absence of OSA, is not accompanied by increased sympathetic activity to muscle vascular beds.

Plasma concentrations of catecholamines were not monitored for several reasons; first, reliable measurements require an indwelling catheter as direct venipuncture may elevate catecholamine levels [14], second, reliable measurements require averaging of at least two blood samples [14], and third, plasma catecholamine concentrations fluctuate and do not reflect the mean rate of norepinephrine released over a longer period of time, such as TST, in contrast to urinary catecholamines [12, 23].

The authors conclude that, in hypertensive males, obstructive sleep apnoea is associated with increased urinary normetanephrine and metanephrine, independent of recognized confounding factors. The finding suggests specific alteration of sympathoadrenal function and metabolic turn-over of catecholamines in obstructive sleep apnoea. This mechanism may, at least in part, explain the association between obstructive sleep apnoea and cardiovascular morbidity.

Acknowledgements. The authors would like to thank U. Spetz-Nyström, the research nurse, for her excellent assistance.

\section{References}

1. Ziegler MG, Nelesen R, Mills P, Ancoli-Israel S, Kennedy B, Dimsdale JE. Sleep apnea, norepinephrinerelease rate, and daytime hypertension. Sleep 1997; 20: 224-231.

2. Lapinski M, Przybylowski $\mathrm{T}$, Lewandowski $\mathrm{J}$, et al. Diurnal blood pressure rhythm and urinary catecholamine excretion in obstructive sleep apnoea and hypertension. J Hypertens 1993; 11: Suppl. 5, S292-S293.

3. Hedner J, Ejnell H, Sellgren J, Hedner T, Wallin G. Is high and fluctuating muscle nerve sympathetic activity in the sleep apnea syndrome of pathogenetic importance for the development of hypertension? J Hypertens 1988; 6: Suppl. 4, S529-S531.

4. Otsuka N, Ohi M, Chin K, et al. Assessment of cardiac sympathetic function with iodine-123-MIBG imaging in obstructive sleep apnea syndrome. $\mathrm{J} \mathrm{Nucl}$ Med 1997; 38: 567-572.

5. Fletcher EC, Bao G, Li R. Renin activity and blood pressure in response to chronic episodic hypoxia. Hypertension 1999; 34: 309-314.

6. Brooks D, Horner RL, Kozar LF, Render-Teixeira CL, Phillipson EA. Obstructive sleep apnea as a cause of systemic hypertension. Evidence from a canine model. J Clin Invest 1997; 99: 106-109.

7. Loredo JS, Ziegler MG, Ancoli-Israel S, Clausen JL, Dimsdale JE. Relationship of arousals from sleep to sympathetic nervous system activity and BP in obstructive sleep apnea. Chest 1999; 116: 655-659.

8. Franklin KA, Nilsson JB, Sahlin C, Näslund U. Sleep apnea and nocturnal angina. Lancet 1995; 345: 10851087.

9. Peker Y, Kraiczi H, Hedner J, Löth S, Johansson Å, Bende M. An independent association between obstructive sleep apnea and coronary artery disease. Eur Respir J 1999; 13: 179-184.

10. Bradley TD. Hemodynamic and sympathoinhibitory effects of nasal CPAP in congestive heart failure. Sleep 1996; 19: S232-S235.

11. Palomäki H, Partinen M, Erkinjuntti T, Kaste M. Snoring, sleep apnea syndrome and stroke. Neurology 1992; 42: Suppl. 6, 75-82.

12. Hedner J, Darpö B, Ejnell H, Carlson J, Caidahl K. Reduction in sympathetic activity after long-term CPAP treatment in sleep apnoea: cardiovascular implications. Eur Respir J 1995; 8: 222-229.

13. Minemura $\mathrm{H}$, Akashiba $\mathrm{T}$, Yamamoto $\mathrm{H}$, Akahoshi $\mathrm{T}$, Kosaka N, Horie T. Acute effects of nasal continuous positive airway pressure on 24-hour blood pressure and catecholamines in patients with obstructive sleep apnea. Intern Med 1998; 37: 10091013.

14. Coy TV, Dimsdale JE, Ancoli-Israel S, Clausen J. Sleep apnoea and sympathetic nervous system activity: a review. J Sleep Res 1996; 5: 42-50.

15. Goldstein DS. Plasma catecholamines and essential hypertension. An analytical review. Hypertension 1983; 5: 86-99.

16. Mancia G, Grassi G, Parati G, Daffonchio A. Evaluating sympathetic activity in human hypertension. J Hypertens 1993; 11: S13-S19.

17. Jennum P, Schultz-Larsen K, Christensen N. Snoring, sympathetic activity and cardiovascular risk factors in a 70 year old population. Eur J Epidemiol 1993; 9: 477-482.

18. Elmasry A, Janson C, Lindberg E, Gislason T, 
Tageldin MA, Boman G. The role of habitual snoring and obesity in the development of diabetes: a 10-year follow-up study in a male population. J Intern Med 2000; 248: 13-20.

19. Lindberg E, Sjöström C, Elmasry A, Janson C. Sleep apnea and snoring in males with hypertension. Am J Respir Crit Care Med 2000; 161: A681.

20. Peaston RT. Routine determination of urinary catecholamines by high performance liquid chromatography with electrochemical detection. J Chromatogr 1988; 424: 263-272.

21. Redline S, Tosteson T, Boucher MA, Millman RP. Measurements of sleep-related breathing disturbances in epidemiologic studies: Assessment of the validity and reproducibility of a portable monitoring device. Chest 1991; 100: 1281-1286.

22. Lindberg E, Elmasry A, Janson A, Gislason T. Reported snoring - does validity differ by age? J Sleep Res 2000; 9: 197-200.

23. Peled N, Greenberg A, Pillar G, Zinder O, Levi N, Lavie P. Contributions of hypoxia and respiratory disturbance index to sympathetic activation and blood pressure in obstructive sleep apnea syndrome. Am J Hypertens 1998; 11: 1284-1289.

24. Forster C. Autonomic nervous system neurotransmitters. In: Kalant H, Roschlau WHE, eds. Principles of Medical Pharmacology. New York, Oxford University Press, 1998; pp. 135-148.

25. Eisenhofer G, Friberg P, Pacak K, et al. Plasma metadrenalines: do they provide useful information about sympatho-adrenal function and catecholamine metabolism. Clin Sci 1995; 88: 533-542.

26. Creveling CR, Hartman BK. Relationships between the cellular localization and the physiological function of catechol-O-methylation. In: Usdin E, Borchardt, Creveling CR, eds. Biochemistry of S-adenosylmethionine and Related Compounds. London, Macmillan, 1982; pp. 479-486.

27. Somers VK, Mark AL, Abboud FM. Sympathetic activation by hypoxia and hypercapnia - implications for sleep apnea. Clin Exp Hypertens 1988; A10: Suppl. 1, 413-422.

28. Dimsdale JE, Coy T, Ancoli-Israel S, Mills P, Clausen J, Ziegler MG. Sympathetic nervous system alteration in sleep apnea. The relative importance of respiratory disturbance, hypoxia, and sleep quality. Chest 1997; 111: 639-642.

29. Narkiewicz K, van de Borne PJH, Cooley RL, Dyken ME, Somers VK. Sympathetic activity in obese subjects with and without obstructive sleep apnea. Circulation 1998; 98: 772-776. 